\title{
La súplica a Hera en el «Poema de los Hermanos» de Safo
}

The prayer to Hera in Sappho's «Brothers Poem»

\section{M. a del Henar Velasco López}

\author{
Universidad de Salamanca \\ hvl@usal.es
}

\begin{abstract}
La revisión del papel desempeñado por Hera como señora de las tormentas en distintos episodios permite situar la súplica de Safo en el «Poema de los Hermanos» en el contexto mítico-religioso de su tiempo. La doble faceta que revelan las actuaciones de la diosa, soberana y protectora de la unidad familiar, ayuda a comprender mejor el trasfondo del texto y esbozar una hipótesis sobre la razón última del ruego que Safo le dirige.
\end{abstract}

Palabras clave: Hera; deidad de las tormentas; navegación; Safo; «Poema de los Hermanos»; Fr. 17; nóstoi.

A fresh look at Hera's special relationship with sea-voyages and storms establishes the mythical and religious background of Sappho's prayer to Hera in the «Brothers Poem». The explanation for her involvement in such situations reveals her double role as goddess of marriage and of sovereignity. This analysis adds to our understanding of Sappho's request.

Key words: Hera; Storm divinity; seafearing; Sappho; «Brothers Poem»; Fr. 17; nóstoi.

\section{La súplica a Hera en el 'Poema de los Hermanos' de Safo}

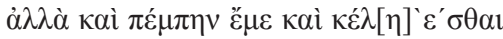

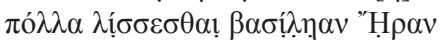

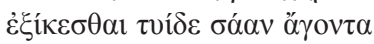

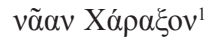

${ }^{1}$ Reproducimos aquí el texto de Obbink 2014, p. 37. A la discusión en distintos foros de Internet que se desencadenó de inmediato en enero de 2014 al comunicarse el hallazgo se sumaron muy pronto nuevas publicaciones: Burris, Fish y Obbink 2014 (trata de otros fragmentos), West 2014 (en concreto el de los Hermanos corresponde a Fr. 9a p. 8 s.), Ferrari 2014. Aspectos más puntuales han sido abordados en ZPE 191 y en otras publicaciones, tan sólo los citaremos en la medida en que interesen para la cuestión aquí abordada. Esta contribución fue aceptada para su publicación varios meses antes de que el libro de Bierl y Lardinois 2016 viera la luz. Agradecemos a J. Rodríguez Somolinos que nos haya facilitado la lectura de dicha obra antes de las últimas pruebas, pues así hemos podido señalar aquellos puntos en que nuestra investigación coincide con alguno de los autores que más recientemente han escrito sobre el tema.

Copyright: (C 2016 CSIC. Este es un artículo de acceso abierto distribuido bajo los términos de la licencia de uso y distribución Creative Commons Attribution (CC-by) España 3.0. 
Mas (conviene) $)^{2}$ que me envíes y me exhortes

a suplicar mucho a Hera soberana

que aquí regrese trayendo, salva,

una nave Caraxo.

Tal es la encarecida súplica dirigida a la reina (basíléan $)^{3}$ Hera para que el hermano de Safo, Caraxo, regrese en una nave intacta ${ }^{4}$. Singular, sin duda. Más si cabe cuando por dos veces en el poema, antes y después, se menciona a Zeus: en la primera estrofa conservada completa para afirmar que él y todos los dioses conocen la verdad de las noticias que a Safo llegan, y en la cuarta para sostener que resultan afortunados y muy dichosos aquellos cuyo sino troca el rey del Olimpo a partir de las penalidades. Además la tercera estrofa afirma que la calma sigue a la tempestad, una referencia que parece cuadrar más a Zeus que a Hera. Pero lo cierto es que a quien dirige sus muchas súplicas es a ella en ésta, la segunda estrofa conservada.

Este punto ofrece un interrogante cuando menos curioso al filólogo clásico: ¿Por qué precisamente a Hera? Veamos si podemos esclarecer un poco la cuestión.

Para empezar, no es la única ocasión en que Safo nombra a la esposa de Zeus en un contexto similar. Incluso la aparición de este nuevo poema permite aquilatar una hipótesis de trabajo que nos habíamos planteado respecto a otro fragmento sáfico, el $17 \mathrm{~V}^{5}$, aquél en que se rememora cómo los Atridas se detuvieron en la isla de Lesbos a su regreso de Troya para suplicar a Hera, Zeus y Dioniso. Por más que se mencione a la tríada, bien conocida también por un poema de Alceo (129 V. $)^{6}$ así como por otras referencias de los antiguos, es clara la preeminencia de Hera ${ }^{7}$ : es la primera

${ }^{2}$ El verbo impersonal está expresado en el tercer verso de la primera estrofa. El segundo infinitivo, según Obbink 2014, p. 43, representa «an original subjuntive or optative in the putative prayer».

${ }^{3}$ Sobre el uso del epíteto, v. Ferrari 2014, p. 2, Obbink 2014, p. 43.

${ }^{4}$ Sobre dicha expresión, v. Nünlist 2014, pp.13-14. Sobre la identidad de quién ha de enviar presumiblemente a una joven Safo a realizar la plegaria se ha sugerido que sea su madre (Obbink 2014 y West 2014, citan los precedentes míticos de Clitemestra-Electra y Helena-Hermíone, también Ferrari), la nodriza (Bettenworth 2015) o Lárico, el hermano pequeño (Stehle 2016, p. 268), Caciagli 2016, p. 436 y Lardinois 2016, p. 183 proponen que sea Eurigio, el tercer hermano de Safo, cabeza temporal de la familia, una vez muerto el padre y dada la ausencia de Caraxo; por un tío materno se inclina Bierl 2016, p.331.

5 También este poema se ha visto enriquecido gracias a los nuevos hallazgos, v. Burris, Fish y Obbink 2014, p. 18 ss., West 2014, p. 3 ss., Ferrari 2014, p. 15 ss., Neri 2014, Caciagli 2016, Nagy 2016.

${ }^{6}$ Pötscher 1987, p. 14 ss.

${ }^{7}$ Nagy 2016 p. 458 aporta un dato interesante: el término $\tau \varepsilon \tilde{\chi} \chi 0 \varsigma \beta \alpha \sigma i ́ \lambda \varepsilon 10 v$ utilizado en Alceo 130a (1. 15) para referirse al recinto sagrado de Messon, escenario también de Safo 17, es glosado como $\tau$ ò $\tau \tilde{\eta} \varsigma^{~ " H}$ Has. En p. 477 sostiene incluso la omnipresencia de Hera. También subraya Boedeker 2016, p. 197 su extraordinaria relevancia. 
deidad mencionada ya en la primera estrofa (verso 2$)^{8}$, a continuación se recuerda el episodio de los Atridas, y de vuelta al presente, pese a lo lacunoso del texto y las diferentes interpretaciones, tanto West (2014, p. 5), como Neri (2014, p. 18 s.), Ferrari (2014 p. 15) $)^{9}$, Obbink (2016 p. 20) y Lidov (2016 p. 423) aceptan la propuesta de Milne que reconstruye el nombre de Hera en el último verso, una invocación que en bella composición de anillo cierra el poema $17 \mathrm{~V}$. Sin embargo, al ser las dos últimas estanzas demasiado fragmentarias, incluso con las nuevas aportaciones, tan sólo podemos imaginar por qué razón acude Safo a Hera.

Se ha supuesto una petición semejante a la de Agamenón y Menelao, esto es, vientos favorables para un viaje desde Lesbos. Al ser tan frecuentes las idas y venidas de las muchachas de su círculo, parece probable, por más que no haya nada en el texto que lo confirme ${ }^{10}$. No hay nada tampoco a favor de otra hipótesis, la que nos formulábamos nosotros en voz baja antes de aparecer este nuevo fragmento, que el viaje atañera a su hermano ${ }^{11}$. Nada hay definitivo y en ese resbaladizo terreno de las conjeturas hemos de dejarlo, pero al menos este nuevo poema pone de relieve que cuando está en juego el ansiado regreso de Caraxo, Safo dirige sus súplicas a Hera.

El poema $17 \mathrm{~V}$. precisamente es único por mostrar una versión del nóstos de los Atridas sin parangón en otras fuentes. El testimonio lesbio ${ }^{12}$ está ligado a uno de los santuarios más famosos de la isla, presumible escenario de sus famosos concursos de belleza y acaso punto clave en la proyección panhelénica de sus poetas ${ }^{13}$.

${ }^{8}$ La reconstrucción de la primera estrofa tras el descubrimiento de los nuevos fragmentos en Lidov 2016.

9 Estos dos autores, Neri y Ferrari, avanzan además lecturas del verso 11, aquél en que el poema vuelve del pasado mítico a la situación presente, en las que se apelaría directamente

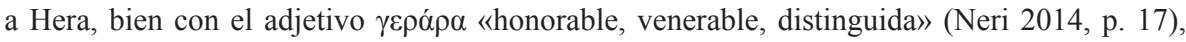
bien con el epíteto cultual $\pi$ ó $v 1 \alpha$ «señora, dueña, soberana» (Ferrari 2014, p. 18).

${ }_{10}$ Page 1970 (1955), p. 62. Referencias a ésta y otras propuestas en Lidov 2004, p. 401 y Neri 2014, p. 13.

${ }^{11}$ Nos congratula comprobar que también Caciagli 2016, pp. 434 y 437 defiende esa idea que ya antes había sugerido. Cf. Obbink 2016, p. 212, n. 20.

12 West 2002.

${ }_{13}$ Alc. 130b V.; Sch. a Il. I 129; Thphr. en Ath. XIII 610a. Cf. Page 1970 (1955), p. 168 y bibliografía citada por Pirenne-Delforge y Pironti 2014, p. 27 n. 6 y 7. También Haug en $R E$ vol. VIII, 1 s. u. Hera p. 378; Calame 2001, p. 98 ss., Neri 2014, Caciagli 2016, p. 433 s. Nagy 2016, p. 458 ss. reconstruye el festival (sacrificio, procesión, súplica, referente mítico, concurso de belleza, representación coral) como contexto de los poemas sáficos, Lidov 2016 sugiere que la fiesta anual incluía tanto la celebración de vuelta a casa de los mercaderes marítimos, como ceremonias matrimoniales y serviría de «performance locus» de los poemas del primer libro de Safo, incluido éste en que se menciona a Caraxo, 
Con estos datos en mente merece la pena preguntarse si constituye también una singularidad sáfica el implorar protección a la reina de los dioses en circunstancias semejantes a la evocada en el Poema de los Hermanos. Creemos que no es así, sino que justamente se inscribe en una tradición bien asentada ${ }^{14}$. Para demostrarlo es vital situar dicha petición en el contexto más amplio de los mitos y la religiosidad helénica, en concreto, revisar el papel de Hera en aquellos relatos que ofrezcan un paralelo válido, que pongan de manifiesto la relación de la diosa con la navegación y las tempestades. Y esto no para reavivar la periclitada cuestión de una Hera, diosa del aire ${ }^{15}$, sino para que el indagar en dichos episodios nos permita entender mejor las razones de su actuación y, por ende, iluminar el ruego de $\mathrm{Safo}^{16}$.

Es éste un examen cuyo desarrollo nos demoraría demasiado. El lector interesado en los detalles de ese análisis podrá seguirlos en otra contribución más minuciosa bajo el título «Hera, señora de las galernas». Aquí nos limitaremos a resumir sus conclusiones prescindiendo de variantes, notas y referencias bibliográficas que allí podrán ser consultadas.

Pasajes míticos como el envío de tormentas contra Heracles a su regreso de la primera guerra de Troya y contra Paris y Helena en su huida, la fabricación de la nube que burla a Ixión o el famoso eidolon de Helena ponen de manifiesto que cuando Hera maneja los fenómenos atmosféricos lo hace con un claro objetivo, la

Boedeker 2016, p. 193 s., aun de acuerdo con la propuesta de Lidov, considera que la situación crítica evocada por Safo en el Poema de los Hermanos exigiría una súplica inmediata, sin esperar a la festividad. Sin embargo, para Lardinois 2016, p.184 no hay garantía de que el poema fuera interpretado allí, sugiere otras alternativas en p. $185 \mathrm{~s}$. Interesante también la aportación de Bierl 2016, p. 305 ss., 323 ss., 335.

${ }^{14}$ Boedeker 2016, p. 190 al reconocer que «in standard Works on Greek religión she [Hera] has little or nothing to do with seafaring and trade» nos reafirma en lo oportuno de nuestra propuesta. En verdad Boedeker señala algunos de los episodios míticos en que protege a héroes en el mar (también Lidov 2004, que sugiere además la situación contraria, el envío de una tormenta a los Atridas en Fr. 17 V.), y propone un interesante recorrido por los santuarios de la diosa localizados en puertos y cabos en el Mediterráneo (también Nagy 2016, p. 473 incide en este aspecto marítimo), pero no llega a analizar esos pasajes ni a buscar una interpretación a la actuación de Hera. Es reconfortante comprobar que planteamientos independientes nos conducen a ambas a conclusiones plenamente coincidentes.

15 Sobre ésta y la otra interpretación enfrentada ya desde la antigüedad de Hera como diosa de la tierra, v. Roscher 1884-1937 (1965), vol. I, col. 2.106 s. Las obras del propio Roscher así como de Welcker y Gruppe allí citadas pueden consultarse cómodamente en Internet Archive.

${ }_{16}$ Pirenne-Delforge y Pironti 2014, p. 28 tan solo hacen una breve mención a esta cuestión, pero, como ellas y como Boedeker, confiamos en matizar la figura de Hera y enriquecer la interpretación del nuevo poema sáfico. 
defensa del matrimonio. Por su parte, lances más breves en los que Hera se sirve del trueno o la bruma para apoyar a Agamenón, los más célebres como la ayuda prestada a Jasón en su paso de las Rocas Planctas y las Errantes y, por el contrario, el despliegue de medios con que dificulta el viaje de Eneas están al servicio de la legitimidad de la soberanía. Mito y rito caminan muchas veces de la mano. Los episodios aludidos se corresponden con referencias textuales y testimonios arqueológicos que inciden en la protección que Hera dispensa en los viajes por mar a larga distancia.

No vamos a entrar aquí en la cuestión de si el dominio que ejerce Hera sobre los fenómenos atmosféricos se debe a la mera asociación con su esposo y hermano, Zeus ${ }^{17}$, o si estamos ante un resquicio que permite atisbar la situación indoeuropea, aquella en la que el dios del cielo y el de la tormenta son dos figuras distintas ${ }^{18}$.

Aquí y ahora lo que nos interesa subrayar es que sus intervenciones responden a un claro objetivo, el perfecto desempeño de las funciones que tiene asignadas en la estructura bien fraguada con la que a nosotros se nos presentan los mitos griegos: Hera, esposa de Zeus, diosa del matrimonio, es también la soberana que protege al rey legítimo (Jasón, los Atridas); a su vez, persigue a quienes la ofenden atentando contra el patrocinio de una u otra faceta (Heracles, Eneas), incluso ambas a la vez (Paris desdeña su belleza y promesa de poder y además comete adulterio). De esa manera la diosa garantiza la unidad familiar y la soberanía.

Tras esta rápida mirada a las funciones y actuaciones de la diosa, volvamos los ojos al poema que nos ocupa. Si no hemos errado en nuestro análisis, si las tempestades, la protección o el impedimento de la navegación son un instrumento en las manos de Hera a favor de la legitimidad, quizás podamos encarar el nuevo fragmento sáfico desde esta perspectiva, el mito como paradigma: al igual que a los héroes a los mortales les conviene granjearse el favor de la diosa y evitar su ira.

Safo impetra el auxilio de Hera «reina, soberana» (basílēan v. 6) para su hermano Caraxo, quien ha pasado a la posteridad por tener una amante en Egipto que disgusta a Safo, una hetera de nombre Dórica para ser más precisos hacia la que Caraxo manifestó un comportamiento tan extravagante e indebido que, al parecer, menoscabó

17 Nagy 2016, p. 477 considera que Hera asume incluso la identidad de su consorte que funciona como «the coefficient of Hera in bringing favorable winds for voyagers at sea» en un papel subordinado a la diosa. Boedeker 2016, p.205 considera que «Hera and Zeus balance each other in the Charaxos song: specific vs. general, approachable vs. distant»

${ }^{18}$ Así sucede fuera de Grecia y Roma. Entre otros West 2007, p. 238 considera que precisamente ésa es la situación original. 
el prestigio social de toda la familia ${ }^{19}$. Cabría apuntar entonces que, a juzgar por los paralelos míticos enumerados, los temores de la hermana podrían abarcar un ámbito más allá de lo que hoy prosaicamente llamaríamos el pronóstico del tiempo. La travesía implica atravesar un mar siempre proceloso ${ }^{20}$, en el que Hera puede causar bien o mal. Con su súplica acaso la poetisa confía en que la reina de los dioses ayude a Caraxo, precisamente porque las relaciones que mantiene con la famosa hetera bien podrían haber desagradado a la diosa, como la disgustaron en su día las de Alejandro y Helena, modelo ésta no de mujer casada, sino de la adulta que expresa la seducción que encarna Afrodita. Por tanto, al rogarle «muchas veces o mucho» lo que pretende Safo es que Hera lo devuelva a su patria, sano y salvo, cualidad que en el poema se aplica a la nave que conduce.

Y, de hecho, así debió conseguirlo, pues otro poema presupone un segundo viaje de Caraxo a Egipto que también inquieta a la hermana. En él (15 V, vv. 9-12; Ferrari 2014, p. 10; Lardinois 2016, p. 171 s. con otras referencias) pide a Cipris que Dórica encuentre áspera a la diosa y no pueda jactarse por segunda vez de que el deseo la alcanzó de nuevo, en otras palabras, que no vuelvan a prosperar los amoríos de la hetera con Caraxo. Ésa es, desde luego, la súplica que corresponde al área de influencia de Afrodita, como la que le dirige en el Poema de los Hermanos ha de responder al ámbito de Hera. El trasfondo mítico aquí apuntado junto con la importancia extraordinaria de la reina de los dioses en Lesbos nos ayudan, al menos, a nosotros a calibrar mejor la súplica de Safo.

Ella, Safo, lo espera, según la propuesta de Whitmarsh 2014, como Penélope a Ulises, mutatis mutandis naturalmente. En verdad no sería descartable esa hipótesis de leer el poema como un juego sobre el más famoso de los nóstoi, cuando preci-

19 Poemas 5, 7 y 15b V., el primero aumentado gracias a los nuevos hallazgos (West 2014, pp. 5-7). Cf. Hdt. II 135, Str. XVII 1.33; Ath. XIII 596b, Ou., Ep. XV 67 ss., Page 1970 (1955), p. 48 ss. Más referencias en Obbink 2014, p. 32 n. 1, el análisis de 5 y 15 en Ferrari 2014, p. 4 ss., más en concreto pp. 9-10 sobre la identificación de Dórica. Sobre los juegos de palabras implícitos en este nombre v. Martin 2016, p. 119. Muy valiosas también las reflexiones de Rauflaub 2016 y la posible conexión con otros fragmentos sáficos en Lardinois 2016, p. $172 \mathrm{~s}$. Caciagli 2016 en su reconstrucción de la audiencia del poema, familiares varones y amigos de Caraxo, tiene muy en cuenta hasta qué punto se han visto afectados por su aventura amorosa p. 441 s., pero en p. 447 no descarta la presencia de «a female hetaireia» que también habría sufrido las consecuencias. Según Martin 2016, p. 124, la identidad y solidaridad del grupo quedaría reforzada por la agresión yámbica, clave en su interpretación del poema.

${ }^{20}$ Recuperado ahora el primer vocablo del fragmento $5 \mathrm{~V}$, merece la pena recordar que en ese poema son las «señoras, venerables, augustas ( $\pi$ ó $v v \imath \imath$ ) Nereidas» las interpeladas para que traigan ileso a Caraxo (Ferrari 2014, pp. 5-6). El Fr. 20, que menciona una tormenta, no ofrece indicios suficientes para relacionarlo con los viajes del hermano de Safo (Ferrari 2014, p. 12, Lardinois 2016, p. 174). 
samente -añadimos nosotros- el fragmento $17 \mathrm{~V}$. revela una versión única del que atañe a los Atridas que también acudieron al templo de la diosa ${ }^{21}$. Eso no invalida el contexto que hemos intentado iluminar para explicar que sea ella, Hera, precisamente la invocada, la diosa que detentaba un puesto tan destacado en el santuario lesbio. Es más, incluso, rizando el rizo, y teniendo en cuenta la mención en la estrofa final del otro hermano, Lárico, y el deseo de que se convierta en un hombre ${ }^{22}$, nos atreveríamos a completar esa hipótesis y señalar que Lárico, el hermano pequeño, es comparable a Telémaco ${ }^{23}$, ambos en el paso de la juventud a la madurez.

De otro lado y sin apartarnos demasiado de ese ámbito familiar reconstruido en clave metafórica, las conclusiones a las que conduce nuestra indagación y que transitan por caminos diferentes a los hollados por Polignac 1993 vienen a darse la mano con las apuntadas por este autor a raíz de su análisis de las ofrendas votivas de barcos a Hera: la diosa protege el universo doméstico, la perpetuación del oikos $^{24}$, garantiza su estabilidad, así como el movimiento del viaje que amplía y enriquece.

${ }^{21}$ El elemento coincidente con la épica homérica es que según el relato de Néstor en Od. III 169 fue en Lesbos donde decidieron la ruta que iban a seguir.

22 Obbink 2014, p. 35. Coincidimos con Boedeker 2016, p. 207 en que la madurez y el matrimonio que presumiblemente aguardan a Lárico (cf. Lardinois 2016, p. 180) son facetas en el área de control de Hera. Cf. Bierl 2016, p. 323, quien subraya que el propio Caraxo debe concluir sus ritos de paso con una boda adecuada en Lesbos, ve en él un modelo para el círculo sáfico del futuro novio (p. 326 s.).

${ }^{23}$ También West 2014, p. 9 s. menciona a Telémaco en relación con Lárico, «inert and submissive, rather like Telemachus at the start of the Odyssey before Athena activates him». Martin 2016, p. 121 ss. acoge esa propuesta, pero acorde con su lectura yámbica del poema le confiere un tono ofensivo. Obbink 2016 p. 212 compara a Caraxo con Ulises (cf. Nünlist 2014, p. 13) y señala otras sugerentes correspondencias: Safo - Penélope, Dórica - Calipso o Circe. Cf. Bierl 2016, p. 310. Stehle 2016 p. 290, convencida de que los hermanos parecen «tipos», prefiere comparar a Lárico con Paris. Lardinois 2016, p. 180 no se muestra de acuerdo con la supuesta crítica a Lárico que sostienen Martin y Stehle, pero sí defiende que tanto él como Caraxo son probablemente caracteres ficticios (p.185). En sus nombres parlantes se fija Bierl 2016, pp. 318, 320.

${ }^{24}$ Igualmente Ferrari 2014, p. 4 insiste en la unidad del hogar que pese a la apertura del mundo femenino, representado en el poema por Safo y verosímilmente su madre, en Lesbos gira en torno al varón: Caraxo, ausente, Lárico, aún menor. Por su parte Boedeker 2016, p. 190 recoge el comentario de Nünlist 2014: la súplica sugiere problemas también en casa, no sólo en el mar, y añade: «Hera is asked to bring about relief and safety for both sides, the voyager and those awaiting him». Para Lardinois 2016, p. 176 las tormentas que amenazan a Caraxo también pueden aludir a las dificultades que atraviesan en casa. Bierl 2016, p. 316 ss. no descarta un uso político metafórico de la nave y propone otros significados simbólicos: amoroso y místico. 
Difícil adivinar qué pensaría de estas conjeturas la «coronada de violetas». ¿Las aprobaría o acaso ladearía la cabeza con infinita dulzura al tiempo que esbozaría una suave sonrisa para compensar, al menos, la tentativa de penetrar en el secreto de esos versos, a través de los cuales estaba segura de que la recordaríamos?

\section{BibliografíA}

Bettenworth, A. 2015: «Sapphos Amme: Ein Beitrag zum neuen Sapphofragment», ZPE 191, pp. 15-19.

Bierl A. 2016: "'All You Need is Love': Some Thoughts on the Structure, Texture, and Meaning of the Brothers Song as well as on Its Relation to the Kypris Song (P. Sapph. Obbink)», en Bierl, A. y Lardinois, A. 2016, pp. 302-336.

Bierl, A. y Lardinois, A. 2016: The Newest Sappho: P. Sapph. Obbink and P. GC inv. 105, Frs. 1-4, Leiden - Boston.

Boedeker, D. 2016: «Hera and the Return of Charaxos», en Bierl, A. y Lardinois, A. 2016, pp. 188-207.

Burris, S.-F., Fish, J. y Obbink, D. 2014: «New Fragments of Book 1 of Sappho», ZPE 189, pp. 1-28.

Caciagli, D. 2016: «Sappho Fragment 17: Whishing Charaxos a Safe Trip?», en Bierl, A. y Lardinois, A. 2016, pp. 424-448.

Calame, C. 2001: Choruses of Young Women in Ancient Greece: Their morphology, Religious Role and Social Functions, Lanham - Boulder - Nueva York - Oxford. Accessible en nueva versión en $<$ http://chs.harvard.edu/CHS/article/display/3853>, cap. 3, p. 98 ss. $<$ http://chs. harvard.edu/CHS/article/display/3743> (05/12/2014).

Ferrari, F. 2014: «Saffo e i suoi fratelli e altri brani del primo libro», ZPE 192, pp. 1-19.

Lardinois, A. 2016: «Sappho's Brothers Song and the Fictionality of Early Greek Lyric Poetry», en Bierl, A. y Lardinois, A. 2016, pp. 167-187.

Lidov, J. 2004: «Hera in Sappho, fr. 17 L-P, V. - and Aeneid I?», Mnemosyne 57, 4, pp. 387-406.

Lidov, J. 2016: «Notes on the First Stanza of Fragment 17», en Bierl, A. y Lardinois, A. 2016, pp. 415-423.

Martin, R. P. 2016: «Sappho Iambist: Abusing the Brother», en Bierl, A. y Lardinois, A. 2016, pp. 110-126.

Nagy, G. 2016: «A Poetics of Sisterly Affect in the Brothers Song and in Other Songs of Sappho», en Bierl, A. y Lardinois, A. 2016, pp. 449-492.

Neri, C. 2014: «A Hoped-for Festival? (Sapph. Fr. 17 V. and P.GC. inv. 105 fr. 2 col. ii 11. 9-28)», Eikasmos 25, pp.11-24.

Nünlist, R. 2014: «Das Schiff soll unversehrt sein, nicht voll! Zu Sapphos neuem Lied über die Brüder», ZPE 191, pp. 13-14.

Obbink, D. 2014: «Two New Poems of Sappho», ZPE 189, pp. 32-49.

Obbink, D. 2016: «Goodbye Family Gloom! The Coming of Charaxos in the Brothers Song», en Bierl, A. y Lardinois, A. 2016, pp. 208-224.

Page, D. 1970 (1955): Sappho and Alcaeus. An Introduction to the Study of Ancient Lesbian Poetry, Oxford. 
Pirenne-Delforge, V. y Pironti, G. 2014: «Héra et Zeus à Lesbos: entre poésie lyrique et décret civique», ZPE 191, pp. 27-31.

Polignac, F. de 1993: «Héra, le navire et la demeure: offrandes, divinité et société en Grèce archaïque» en De la Grenière, J. (dir.), Héra. Images, espaces, cultes. Actes du Colloque International du Centre de Recherches Archéologiques de l'Université de Lille III et de l'Association P.R.A.C. Lille, 29-30 novembre 1993 <http://books.openedition.org/ efr/1338> (05/12/2014).

Pötscher, W. 1987: Hera. Eine Strukturanalyse im Vergleich mit Athena, Stuttgart.

Rauflaub, K. A. 2016: «The Newest Sappho and Archaic Greek-Near Eastern Interactions», en Bierl, A. y Lardinois, A. 2016, pp. 127-147.

$R E$ = Wissowa, G. 1893-1980 : Paulys Real-Encyclopädie der classischen Altertumwissenschaft, Stuttgart.

Roscher, W. H. 1884-1937 (1965): Ausführliches Lexikon der griechischen und römischen Mythologie, I-VII, Leipzig, reimpr. Hildesheim.

Stehle, E. 2016: «Larichos in the Brothers Poem: Sappho Speaks Truth to the Wine-Pourer», en Bierl, A. y Lardinois, A. 2016, pp. 266-292.

West, M. L. 2002: «The View from Lesbos», en Reichel, M. y Rengakos, A. (eds.), Epea Pteroenta. Beiträge zur Homerforschung. Festschrift für Wolfgang Kullmann zum 75. Geburstag, Stuttgart, pp. 207-219 (recogido en Hellenica. Selected Papers on Greek Literature and Thought, vol. I: Epic, Oxford 2011, pp. 392-407).

West, M. L. 2007: Indo-European Poetry and Myth, Oxford.

West, M. L. 2014: «Nine poems of Sappho», ZPE 191, pp. 1-12.

Whitmarsh, T. 2014: «Sappho sings again», <http://www.huffingtonpost.co.uk/tim-whitmarsh/ sappho-poetry_b_4691297.html> (05/11/2014).

Fecha de recepción de la primera version del artículo: 01/06/2015

Fecha de aceptación: 06/07/2015

Fecha de recepción de la version definitiva: 05/02/2016 